\title{
The Surviving Sepsis Campaign: research priorities for the administration, epidemiology, scoring and identification of sepsis
}

\author{
Mark E. Nunnally ${ }^{1 *}$ (D) Ricard Ferrer ${ }^{2,3}$, Greg S. Martin ${ }^{4}$, Ignacio Martin-Loeches ${ }^{5,6}$, Flavia R. Machado ${ }^{7}$, \\ Daniel De Backer ${ }^{8}$, Craig M. Coopersmith ${ }^{9}$, Clifford S. Deutschman ${ }^{10,11}$ and For the Surviving Sepsis Campaign \\ Reasearch Committee
}

\author{
${ }^{*}$ Correspondence: \\ mark.nunnally@nyulangone.org \\ ${ }^{1}$ New York University School \\ of Medicine, New York, NY, USA \\ Full list of author information is \\ available at the end of the article
}

\begin{abstract}
Objective: To identify priorities for administrative, epidemiologic and diagnostic research in sepsis.

Design: As a follow-up to a previous consensus statement about sepsis research, members of the Surviving Sepsis Campaign Research Committee, representing the European Society of Intensive Care Medicine and the Society of Critical Care Medicine addressed six questions regarding care delivery, epidemiology, organ dysfunction, screening, identification of septic shock, and information that can predict outcomes in sepsis.

Methods: Six questions from the Scoring/Identification and Administration sections of the original Research Priorities publication were explored in greater detail to better examine the knowledge gaps and rationales for questions that were previously identified through a consensus process.

Results: The document provides a framework for priorities in research to address the following questions: (1) What is the optimal model of delivering sepsis care?; (2) What is the epidemiology of sepsis susceptibility and response to treatment?; (3) What information identifies organ dysfunction?; (4) How can we screen for sepsis in various settings?; (5) How do we identify septic shock?; and (6) What in-hospital clinical information is associated with important outcomes in patients with sepsis?

Conclusions: There is substantial knowledge of sepsis epidemiology and ways to identify and treat sepsis patients, but many gaps remain. Areas of uncertainty identified in this manuscript can help prioritize initiatives to improve an understanding of individual patient and demographic heterogeneity with sepsis and septic shock, biomarkers and accurate patient identification, organ dysfunction, and ways to improve sepsis care.
\end{abstract}

Keywords: Epidemiology, Organ dysfunction, Outcomes, Priorities, Research, Screening, Sepsis, Septic shock, Surviving Sepsis Campaign author(s) and the source, provide a link to the Creative Commons licence, and indicate if changes were made. The images or other third party material in this article are included in the article's Creative Commons licence, unless indicated otherwise in a credit line to the material. If material is not included in the article's Creative Commons licence and your intended use is not permitted by statutory regulation or exceeds the permitted use, you will need to obtain permission directly from the copyright holder. To view a copy of this licence, visit http:// creativecommons.org/licenses/by/4.0/. 


\section{Introduction}

As a consequence of the findings from the methodologies used by the Sepsis-3 Task Force and the 2016 Surviving Sepsis Campaign Guidelines [1], a Research Committee was charged with describing the state of knowledge and setting research priorities for sepsis by the Society of Critical Care Medicine (SCCM) and The European Society of Intensive Care Medicine (ESICM). The joint publication "Surviving Sepsis Campaign: research priorities for sepsis and septic shock" described the output of Delphi process deliberations [2]. The initial manuscript was meant to be followed by subsequent articles expounding on key areas of interest. Among these were administrative concerns, epidemiology and the scoring and identification of sepsis.

\section{Methods}

As part of a modified Delphi consensus process, members of the Research Committee deliberated research questions to be prioritized. Each member initially submitted five research questions on whatever sepsis topic they thought most important. Through a voting prioritization process [2], these questions were reduced and subsequently categorized. An initial 88 questions were reduced to 22 final clinical questions. Subcommittees were tasked with elaborating on their initial contributions, and are previously published [3, 4]. Another document specifically addresses research priorities for Coronavirus Disease 2019 [5]. For this manuscript, subcommittees were tasked with generating expanded reviews of remaining questions. Two questions addressed administration and epidemiology, and four questions addressed scoring and identification. For efficiencies, the two subcommittees were combined and charged with producing a single manuscript.

Each question was assigned to a subcommittee member for further elaboration. Each elaborating author was asked to provide further background to the research question by addressing what is known, what is not known/gaps in knowledge, and provide future directions for research priorities. These contributions were circulated among the group and harmonized into a single document. Editing was managed by all members and further input given by the committee co-chairs. We present the results of this process. The findings presented pertain to the adult sepsis guidelines, but many be relevant to pediatric sepsis too.

\section{Results}

\section{What is the optimal model of delivering sepsis care?}

The Surviving Sepsis Campaign supports a structured approach to sepsis care that is based on process measures and care bundles. Such structure might raise awareness about the problem and provide a consensus approach to its diagnosis and treatment, emphasizing measures that are supported by existing science. To realize the guidelines' full potential, science supporting the recommendations must clarify not only the benefits and risks of individual interventions, but how these interventions, in combination and applied in a complex clinical environment, improve the process of care to realize better outcomes. 


\section{What is known}

Sepsis is a time-dependent condition in which hourly delays in diagnosis or in the initiation of effective treatments are associated with adverse outcomes and higher costs [6-10]. Unfortunately, the early identification of sepsis among patients is challenging $[11,12]$. Sepsis improvement programs exist to mitigate difficulties in diagnosis, aid the implementation of evidence and improve sepsis care and clinical outcomes.

Education about best practices in sepsis has benefitted the clinical approach to patient care [13]. Irrespective of the setting of presentation, sepsis performance improvement programs lead to improved outcomes. Data suggest that programs work by enriching clinical judgment [14-16]. Importantly, sepsis education programs improved patient survival [17]. Programs focused on early detection, timely administration of antibiotics, control of infection, appropriate initial resuscitation, bundle compliance, critical task completion, and achievement of important endpoints [18-23]. Comprehensive approaches to provide sepsis education to clinicians of every specialty, especially nurses, advanced practice providers and physicians, are among the demonstrably effective strategies [17]. Of particular value were approaches such as e-learning sepsis modules, reference pocket guides, and posters containing sepsis bundles and antibiotic algorithms.

Best practices and goals are sometimes controversial, and incentivizing timely implementation can introduce trade-offs. For example, improvements associated with the early provision of antibiotics may increase the risk of administering the wrong drug [24]. The setting also matters. Prehospital antibiotics for suspected sepsis cases were not clearly associated with better outcomes, although they have not been studied for more severe sepsis presentations [25].

Mandatory quality improvement programs improved sepsis bundle compliance and enhanced sepsis care [9, 26-29]. However, these models of care delivery raise concerns about the relative importance of clinical judgment versus standardized care. Studies assessing physician adherence to inflexible quality metrics suggested that flexibility should be allowed [30]. Data indicate that performance incentive policies should focus on learning (e.g., sharing of best practices, improving the workflow of care processes) [31]. The evidence suggests that education-based quality improvement approaches might enhance the willingness of healthcare professionals to adopt sepsis performance improvement programs.

Locally directed protocols that used standard criteria and outcomes enhanced multiprofessional involvement and coordination [13] and improved efficiencies and outcomes, establishing unified management criteria [32,33]. In contrast to assigning responsibility to individual groups of clinicians, team-based strategies facilitated process management across specialties, ensuring continuity of care, adequate coordination, and staff inclusion to achieve measurable endpoints [34]. Time to intervention is often emphasized as a metric for improvement [35], and early antibiotics correlated with improved survival in septic shock [36]. Sepsis bundles, based on educational programs, were created to standardize care, avoid missed treatment opportunities, and enhance awareness [37]. Implementation of bundles correlated with improved outcomes [38].

Ineffective communication and coordination of care may lead to adverse consequences and patient harm. Medical lapses, misadventures, delays, conflicting information, repeated tests, polypharmacy, higher costs, increased workload, and 
reduced confidence in decisions can result from suboptimal professional interactions, either through uncoordinated activity or poor shared situational awareness [39-42]. Structured written communications, enhancing work flows and feedback have been proposed as methods to improve the process of communication in healthcare [34].

Key points:

- Timely application of appropriate therapies is associated with improved outcomes in sepsis care.

- Improvement programs can have unwanted effects, such as the delivery of unnecessary therapies when the diagnosis is uncertain.

- Effectiveness of quality improvement programs correlates with enhancing clinical judgment, speeding delivery of care, establishing clear responsibilities, and facilitating coordination between clinicians.

\section{What is not known/gaps in knowledge}

Although bundles are associated with improved outcomes, there is limited understanding about sources of effectiveness or how to test them. Key questions exist for the roles of coordination, teamwork and group cognition. An important knowledge gap concerns the roles for standardization and flexibility to account for local and patient-specific conditions. Endpoints, their measurement, and how results influence performance need further explanation.

A knowledge gap exists regarding strategies for health care modeling in different environments. Educational research is lacking to define and address gaps in understanding about best practices. Using care standardization as an endpoint requires ongoing measurement against an evolving evidence base, as standards and best practices change over time. Local factors that might drive variability are inadequately described. How knowledge gaps lead to variability in implementation of sepsis care is largely unknown.

Current detection strategies emphasize rapid identification of patients at risk of further clinical deterioration. The time-dependence of detection and interventions could be better described. Uncertainty in sepsis research investigating the timing of care delivery relates to an inconsistent approach to "time zero" for therapies. Controversies about timing trade-offs for therapies such as antibiotics persist, and are informed by limited data that illustrate either risks or benefits, but not both. Tradeoffs may vary by location and situation.

Studies of out-of-hospital antibiotic administration do not adequately address severity of illness, which might influence the benefits of timely administration. Although protocols are appealing to standardize care, they require validation, due to a lack of understanding of the factors that make them optimal [43-45]. The clinical community still faces a gap in the science of protocol application in a complex environment. Relatively little is known about pre-hospital emergency care [46]. There is ongoing need for better indicators for triage of critical care resources [47]. 


\section{Future directions}

Improvements in the administration of sepsis care will come from a stronger foundation in education, organizational and cognitive sciences. Cost-effectiveness, delivery efficiencies and unwanted trade-offs should be compared. Means of improving situational awareness, interdisciplinary collaboration and communication are objectives for investigation. Cognitive and social research exploring interventions that enhance collaboration could provide useful information for the creation of more effective quality improvement strategies. Identifying key metrics, ways to measure them and provide feedback to participants might improve program effectiveness. As more effective programs result from such investigations, another future target for systems research should be a better understanding of how to enhance the willingness of healthcare professionals to adopt sepsis performance improvement programs.

Various cognitive artifacts, objects or cues that help clinicians think, might improve group consensus and sense-making. Sepsis bundles were developed as a cognitive strategy for implementing guideline recommendations, and future efforts are needed to build a better working knowledge of how bundles work and how to improve upon them to facilitate better care. Electronic medical record data management and alerting could support the early detection and efficient and timely delivery of high-quality interventions to patients with sepsis $[15,48,49]$. Artificial intelligence/machine learning tools may facilitate the processing of large amounts of data to enhance detection and treatment systems.

\section{Important research questions:}

- What impacts, positive and negative, occur as a consequence of standardized sepsis care, taking into consideration timing, appropriateness (e.g., missed diagnosis) and effectiveness?

- What interventions enhance coordination and communication, and how can these activities be measured?

- What devices can help clinicians manage complex decision-making in the setting of sepsis?

\section{What is the epidemiology of sepsis susceptibility and response to treatment?}

A goal of epidemiology is to infer biological rationales from population-based observations [50]. In sepsis research, epidemiology would help advance the understanding about how population data influence sepsis susceptibility, clinical course and response to therapy. Many factors play roles, including age, comorbidities and access to health care. When identified and isolated, the effects of these independent variables on relevant outcomes can be tested. Challenges include the dominance of one independent factor over others and variability in measurement. Other concerns relate to definitions and the relationship between biologic and population data when assessing prognostic/diagnostic performance. 


\section{What is known}

Applying different sepsis definitions leads to variable assessments of sepsis epidemiology. For example, while overlap is quite high [51], Sepsis-2 definitions [52, 53] identify some patients not identified when Sepsis-3 [54] criteria are applied, and vice-versa. Similarly, different data sources affect estimates of the incidence and mortality [55]. Cause-of death-based determinations suggest that the global incidence of and mortality from sepsis are declining [56]. These same data attribute $20 \%$ of all deaths to sepsis, and indicate that $85 \%$ occur in lower- and middle-income countries, highlighting ongoing disparities and opportunities for research [57]. In contrast, analyses using clinical data suggest that the incidence of sepsis and the number of sepsis-related deaths have remained relatively static over time. Healthcare claims-based data, which are limited to the United States, indicate variability $[55,58-60]$. Post hoc analysis indicates that approximately $30 \%$ of patients with a sepsis-like syndrome have a "possible" likelihood of infection [61]. Even in highincome countries, racial and gender disparities affect the risk of sepsis and mortality. Higher rates of comorbidities in some populations confound analyses of disparities [62]. Intra-country causes of disparities include structural inequities, socio-cultural factors and differences common across high-, middle- and low-income countries [59].

Using epidemiologic data like gender, ethnicity, nationality, resource availability, age, and comorbid conditions in sepsis research is difficult, in large part because of confusion arising in the criteria used to link outcomes to independent clinical variables. For example, the effects of age or coexisting illness on outcome can overwhelm the contribution of other variables. One approach to this problem is to use available data to create sepsis "subtypes". Clinical presentation, progression, response to treatment, and risk of mortality may be affected by variations in the response of each unique host [63-65]. Biomarker-based strategies identified heterogeneity in treatment effects in sepsis, and may improve ways to assess therapies in better-selected research populations [66-69] or improve the diagnosis of infection [70]. Although reliant on molecular and pathobiology-driven characteristics, phenotypic models could enrich classic epidemiologic data [71-75]. Four sepsis clinical phenotypes correlated with clinical outcomes and host immune-response patterns [75]. Mortality in one group, which included patients who were older and sicker, was higher than in the other three. Using computer modeling to alter the characteristics of patients included in several highly cited clinical trials resulted in outcomes that differed significantly from what was observed in the actual trial. Sepsis response signatures or Molecular Diagnosis and Risk Stratification of Sepsis (MARS) clusters are another model to identify specific clinical phenotypes $[72,73,76]$.

Key points:

- Changing sepsis criteria lead to varying estimates of incidence and mortality.

- The prevalence of comorbidities and access to health care resources in different populations contribute to different estimates in sepsis epidemiology,

- Creation of "types" using multiple data sources enhances diagnosis and prognosis estimates. 


\section{What is not known/gaps in knowledge}

The lack of consensus on definitions/clinical criteria and on the appropriate source of data makes quantifying the global burden of sepsis challenging [77]. Confirmation of infection is also imprecise. While a number of studies have compared different approaches to data acquisition and different diagnostic criteria, parsing the effects of covariates such as age and comorbidity from one another is difficult. Given the lack of effective markers, different studies may address different populations. Study settings and methodologies are key factors in variability. Comparing data from low-resource and high-resource areas is problematic because of differences in infecting organisms, implementation of proven treatment approaches and access to critical supplies and personnel [77]. These circumstances preclude extrapolation between settings [57]. Selecting patients for studies based on the severity of disease does not account for specific host responses and other undescribed heterogeneous variables. Comparisons between studies are difficult because the contributions of pathogen- and host-specific factors on clinical progression and outcomes are not sufficiently accounted for by most commonly collected epidemiologic data. We lack adequate models to classify and select patients to evaluate specific treatments or allocate resources. Currently there is no validated means, outside of infection-specific therapies (antibiotics, source control) to individualize treatments based on patient subtypes [78].

Until definitive classification criteria are developed, estimating epidemiologic trends and identifying appropriate endpoints will remain challenging. Classifying sepsis on the basis of clinical or molecular phenotypes/endotypes must be refined if the classifications are to identify high-risk patients or evaluate therapies [79]. In spite of decades of research, biomarker-based strategies have provided neither diagnostic specificity nor identified more precise entry criteria for clinical trials [68, 71, 79]. Absent the ability to alter a biomarker or phenotype and observe a change in a biologically relevant outcome, or development of a database so large that genetic incongruities become statistically irrelevant, biomarker- or phenotype-based approaches are of limited value. Much needs to be learned about the effects of comorbid conditions on sepsis [80-82].

\section{Future directions}

In order to better apply population data to sepsis research, further efforts must be made to disentangle the effects of multiple independent variables on susceptibility and treatment responses. Isolating the effects of gender, race/ethnicity, nationality, and resource availability should be an explicit goal of future epidemiologic and therapeutic investigations. Investigations should determine if separating patients into subgroups could be useful earlier in the disease, affecting prognostic prediction, use of ICU resources, or guiding treatment $[47,83]$. Prognostic enrichment should be tested to improve the suitability of patients to receive risky therapies and to refine enrollment in clinical trials [84]. Biological evidence may become a component of a sepsis definition and reduce heterogeneity if they can enhance population data [83, 85]. Model derivation, validation and application to published and proposed studies should become a component of trial design and analysis. Efforts should explore 
accruing larger datasets to account for genetic and other variabilities among patients and fit a model that accounts for major confounders, which themselves should be better characterized.

\section{Important research questions:}

- How can investigators disentangle complex interactions to isolate important clinical factors when investigating sepsis therapies?

- What factors identify subgroups for diagnostic or prognostic enrichment of study cohorts?

- How can datasets be managed to reduce confounding or the dominance of one factor over other important ones when trying to describe sepsis subsets?

\section{What information identifies organ dysfunction?}

Sepsis is defined as "life-threatening organ dysfunction caused by a dysregulated host response to infection" [55]. Thus, organ dysfunction is one of the three defining characteristics of the disorder. Identification of organ dysfunction is particularly important because it helps address a key concern-the absence of "gold standard" criteria that definitively demonstrate that sepsis is present. It thus becomes necessary to use a surrogate or proxy for sepsis. In many large epidemiologic studies, patients are assumed to have sepsis if they meet the test of "outcomes validity"-that is, if they are infected and arrive at some outcome consistent with sepsis. Most commonly, this outcome is death. Other examples of outcomes validity include a substantial ICU length of stay or a need for mechanical ventilation. Unfortunately, outcomes validity is rarely useful at the bedside. The clinician caring for a patient suspected of having sepsis wants to know long before most viable outcomes have been reached. The alternative approach is "construct validity". The proxy for sepsis under this approach is that the patient develops a state that "looks like sepsis". Construct validity is highly subjective. The ability to use organ dysfunction, a defining characteristic of sepsis, as a construct substantially removes some subjectivity, and therefore is desirable.

At times, it is difficult to identify organ dysfunction, or to differentiate it from the other defining characteristics of sepsis, infection and a dysregulated host response. For example, organ dysfunction may be dependent on the host response, and dysregulation of the host response may in turn depend on the infecting organism. Further complicating matters is terminology; dysfunction, injury and failure are often used interchangeably. For convenience, failure may be thought of as an irreversible process, while dysfunction denotes the possibility that the process can be reversed [86-88]. However, distinguishing organ dysfunction from organ injury may be more complicated because identification of both may depend on the use of another set of proxy measures-biomarkers.

\section{What is known}

Biomarkers for sepsis-induced dysfunction have been validated for several organ systems $[71,89,90]$. In rare cases, clinical dysfunction can be identified without requiring some marker of injury. Decreased myocardial contractility, most often identified using 
echocardiography, indicates myocaridal dysfunction but measuring troponin is rarely useful. More often clinical criteria for a sepsis-induced abnormality rely on biomarkers that correlate with dysfunction and/or injury [91-94]. For example, the effects of sepsis on the liver are characterized by hyperbilirubinemia and impaired clotting studies (dysfunction of bile acid transport and protein synthesis, respectively) and elevated serum levels of transaminases, indicative of hepatocellular injury.

Objective criteria exist to denote lung and kidney dysfunction $[89,90]$. However, biomarkers that indicate dysfunction alone (hypoxemia, elevated creatinine) may be nonspecific and may occur late in the course of sepsis. More sensitive markers most often reflect injury (altered pulmonary compliance, abnormal chest radiography, elevated levels of KIM-1). Use of inflammatory biomarkers in patients with the acute respiratory distress syndrome (ARDS) has identified subsets ("phenotypes") that correlate with outcomes such as response to fluids, positive end expiratory pressure (PEEP) or certain drugs [95-99]. Biomarkers for gastrointestinal, brain, peripheral nerve or skeletal muscle dysfunction and/or injury are nonspecific. Some combinations of biomarkers related to dysfunction in different organ systems have prognostic value. These include the Sequential Organ Failure Assessment (SOFA) and Multiple Organ Dysfunction (MODS) scores [100-104].

Key points:

- Many markers for organ dysfunction are nonspecific.

- Combinations of markers may improve the ability to detect organ dysfunction.

- Dysfunction and injury are separate processes, but frequently confused.

\section{What is not known/gaps in knowledge}

In most cases, the relationship between most biomarkers and actual organ dysfunction has remained elusive. The deficit in knowledge reflects the absence of gold standards, and is also a comment on the lack of studies truly validating many biomarkers. Pathobiology of organ dysfunction is not well described. In addition, the descriptors of organ dysfunction change as sepsis progresses. The relationship between dysfunction and injury in specific organ systems is poorly characterized. When testing and applying criteria for organ dysfunction, investigators and clinicians need to account for changes that reflect infection itself versus the effects of other pathologic states (e.g., shock) or insults (e.g., nephrotoxins). Counting dysfunction by organ systems does not adequately explain the concept of sepsis-associated dysfunction, nor does it explain the interrelations between different dysfunctions.

\section{Future directions}

In the future, clinical criteria for sepsis should be informed by patterns that reflect a disease state with expected outcomes whose trajectory can be corrected with proper therapies. Clinicians and researchers should disentangle dysfunction caused by new-onset sepsis, by sepsis perturbations (e.g., shock) or by pre-existing sepsis. A better approach to melding indices of cell damage with those of impaired organ function is fundamental. Efforts are needed to explore the relationship between mechanisms of injury and 
dysfunction. For example, both microcirculation changes and mitochondrial aberrancies are invoked as causes of injury. It is likely, however, that both are induced by sepsis, and their relationship to each other is unclear. Therapeutic targets of interest include prognosis markers and correction of organ dysfunction. Investigators should look for better markers that can provide actionable information before injury has already developed, and indicate a process whose culmination is sepsis-induced organ dysfunction. A key gap in understanding the pathobiology of sepsis could be addressed by efforts to integrate measureable data qualifying function and dysfunction.

A major obstacle to research is circular logic. By defining sepsis as organ dysfunction from a dysregulated response to infection but lacking a way to establish that dysfunction is sepsis-related (because sepsis is defined by organ dysfunction in the first place), clinicians and researchers constantly contend with establishing which came first. The proposed paths we have cited offer an opportunity to solve this problem and conceive of interventions to prevent further morbidity.

Understanding the ways dysfunction can be detected will aid in identifying patients for therapies. Integrating studies that measure and combine imaging, molecules and assess function into more specific patterns could help fulfill the goal of establishing validated signals of how a dysregulated response evolves. Investigation of phenotypic variation might yield useful information about individual susceptibilities to dysfunction, or help determine patterns specific to sepsis. All investigations must contend with the reality that sepsis outcomes depend on patients' responses, comorbid conditions and baseline status, sources of infection and appropriateness of therapies. Being able to measure function and dysfunction is a way to disentangle these variables and a route to therapeutic insights.

\section{Important research questions:}

- What is the pathobiology of organ dysfunction?

- What is the relationship between dysfunction and injury?

- How can dysfunction from sepsis be differentiated from that caused by other coexisting pathologic states (e.g., shock, toxic drugs)?

\section{How can we screen for sepsis in varied settings?}

While epidemiologic study helps determine sepsis susceptibility and response to therapy in populations, insights can be used to identify patients for clinical care. Better description and categorization of patients supports clinical diagnosis and effective therapies. A lack of clinical criteria that effectively screen for sepsis is a major challenge. This defect has repercussions for epidemiology, treatment, quality improvement initiatives and research efforts.

\section{What is known}

Absent a diagnostic gold standard, outcome proxies such as mortality or ICU admission are generally used to identify patients with sepsis. These proxies calibrate a screening model, but they can be influenced by factors such as standards of care, 
setting and inequities. For Sepsis-3, using a SOFA score leverages available but imperfect proxies for organ dysfunction as criteria for sepsis [102]. Improvements in sensitivity, specificity, positive and negative predictive values, availability, resource consumption and performance for individual patients and populations are important to assess new screening tools.

Elements of the systemic inflammatory response syndrome (SIRS) criteria [105] have been used in previous sepsis definitions and in many clinical screening programs. While these criteria are appealingly simple, they do not account for a key component of sepsis-organ dysfunction. Indeed, the original paper advocating for the use of SIRS criteria recognized this problem by inventing a new term-severe sepsis-to include organ dysfunction [52, 105]. Evidence demonstrating that SIRS criteria are accurate or valid indicators of sepsis is limited $[9,106]$.

The Sepsis-3 Task Force criteria propose the quick Sepsis-Related Organ Failure Assessment (qSOFA) score as a prompt for clinicians to look for underlying organ dysfunction and sepsis in non-ICU patients $[54,55]$. Predictive validity for mortality was better than SIRS and comparable to SOFA scores in the patient population examined. Studies in high- and middle-income settings demonstrated a low sensitivity for the outcomes of mortality and the receipt of critical care interventions $[107,108]$, and suggested low specificity [109] as a screening tool for sepsis. These studies did not investigate qSOFA as a prompt for further clinical assessment. Other studies support the validation of the qSOFA criteria as a screen, but similarly do not study the score in the context intended by its creators [110-113].

Using the full SOFA score as a screening tool is appealing in that it incorporates the concept of organ dysfunction, albeit in a limited way. Derivation and validation studies suggest it is useful in the ICU setting and that an increase in score of 2 or more is highly predictive of hospital mortality [103]. The eSOFA score is a simplified version optimized for electronic health records. In a small cohort of patients, eSOFA's performance was better than the Sepsis-3 SOFA criteria [114]. The score uses widely available criteria and is practical to use. eSOFA may be best suited for use in retrospective review, as it negates many of the biases inherent in administrative databases.

Clinical deterioration models and early warning scores, such as the National Early Warning Score (NEWS) or the Modified Early Warning Score (MEWS) [115, 116], may predict deterioration in inpatients and assist in ED triage, but they are operationally dependent on the sensitivity cutoff point used [117, 118]. An important consideration in implementing a warning system is tuning it to local conditions in order to balance excessive alarms with delays in diagnosis. Of note, these scores are not specific for sepsis.

In resource-limited settings, screening is mostly based on available data and cannot count on costly diagnostics or the deployment of response teams. The routine monitoring of vital signs and vigilance for infection can raise suspicion of sepsis, but vital sign abnormalities can be seen in many non-sepsis conditions [119].

Predictive analytics can currently produce robust, adaptive models to sort patients by risk for various outcomes, and models exist to predict outcomes highly correlated with sepsis [120-122]. To be effective, these models must optimize performance, make timely diagnoses, and be linked to actions that improve outcomes. The 
analytic systems are the afferent arm to prompt timely interventions. Like warning systems, these models must minimize unnecessary alerts that overwhelm clinicians and resources.

Rapid acquisition of actionable data is crucial. Screening too early erodes specificity. Screening too late reduces the ability to mitigate deterioration and organ dysfunction. The original qSOFA validation study considered data from $48 \mathrm{~h}$ before to $24 \mathrm{~h}$ after the onset of infection [54]. This period of time remains the best described window of information, but may change based on new ways to measure dysfunction. Table 1 lists several scoring systems.

Key points:

- Sepsis identification is based on proxies for dysfunction that are used in combination (e.g., SOFA score).

- qSOFA was conceived as a quick tool to prompt further clinical assessment for sepsis.

- Warning systems may enhance sepsis screening, but must be tuned to optimize alarm volume and effectiveness. These factors can be setting-dependent.

Table 1 Scoring systems used for patients with sepsis and the components that contribute to them

\begin{tabular}{|c|c|}
\hline Score & Components \\
\hline Systemic inflammatory response syndrome criteria & $\begin{array}{l}\text { Temperature } \\
\text { Heart rate } \\
\text { Respiratory rate } \\
\mathrm{PaCO}_{2} \\
\text { White blood cell count } \\
\% \text { immature neutrophils }\end{array}$ \\
\hline Sequential Organ Failure Assessment (SOFA) Score & $\begin{array}{l}\mathrm{PaO} 2 / \mathrm{FiO} 2 \\
\text { Glasgow Coma Score } \\
\text { Mean arterial pressure } \\
\text { Use of dopamine, dobutamine, epinephrine } \\
\text { or norepinephrine, and dose } \\
\text { Bilirubin } \\
\text { Platelet count } \\
\text { Creatinine } \\
\text { Urine output }\end{array}$ \\
\hline Quick Sepsis-Related Organ Failure Assessment (qSOFA) & $\begin{array}{l}\text { Systolic blood pressure } \\
\text { Respiratory rate } \\
\text { Glasgow Coma Score }\end{array}$ \\
\hline National Early Warning Score (NEWS) & $\begin{array}{l}\text { Respiratory rate } \\
\text { Oxygen saturation } \\
\text { Supplemental oxygen } \\
\text { Temperature } \\
\text { Systolic blood pressure } \\
\text { Heart rate } \\
\text { Alert, Voice, Pain Unresponsive (AVPU) Score }\end{array}$ \\
\hline Modified Early Warning Score (MEWS) & $\begin{array}{l}\text { Systolic blood pressure } \\
\text { Heart rate } \\
\text { Respiratory rate } \\
\text { Temperature } \\
\text { AVPU Score }\end{array}$ \\
\hline $\begin{array}{l}\text { Sepsis-Related Organ Failure Assessment optimized for electronic } \\
\text { health records (eSOFA) }\end{array}$ & $\begin{array}{l}\text { Vasopressor initiation } \\
\text { Mechanical ventilation initiation } \\
\text { Creatinine } \\
\text { Bilirubin } \\
\text { Platelet count } \\
\text { Lactate }\end{array}$ \\
\hline
\end{tabular}




\section{What is not known/gaps in knowledge}

The best screening tool for each setting has not been determined. SOFA was developed in cohorts receiving care in high-resource environments, mostly in the United States, and may not perform as well elsewhere. The scale includes outdated therapies and measurements that may not correlate well with true organ dysfunction. An aggregate SOFA score of 2 might represent mild abnormalities in 2 organ systems or more significant abnormalities in one. It is unclear that these are equivalent states in the setting of infection. Existing proxies for organ dysfunction are limited. Linkages to important outcomes not influenced by other external variables are lacking. There are few studies of sepsis screening that take into account the mechanics of how the screening affects work flow and how the work flow changes outcomes. Currently, there is an opportunity to better harmonize definitions, diagnostics and treatments to improve sepsis outcomes. Tradeoffs in different approaches are not characterized, in particular those involving sensitivity and false positive rates, model consistency versus generalizability, detection-focused versus action-focused constructs, and targeting early cases versus all cases.

Until better markers for organ dysfunction are identified and validated, screening for sepsis based on organ dysfunction will be limited. Suspecting infection is still a good first step in screening, but there are currently limited tools to enhance the suspicion that infection is present. Although the calculation of a SOFA score as a screening tool appears reasonable, it lacks prospective validation of its usefulness at the bedside. Deterioration models currently do not use markers of deterioration that are unique to sepsis. Predictive analytics systems have performed adequately in detecting sepsis cases retrospectively, but there is no clear demonstration that their use effectively changes relevant clinical outcomes.

\section{Future directions}

Better screening for sepsis would include improved screening for organ dysfunction. Selecting better proxies for organ dysfunction and, more problematically, for a dysregulated host response, and validating their predictive abilities in a variety of settings are imperatives for improving screening. These proxies would ideally be easily available, capture dysfunctions early and be specific for sepsis-induced organ dysfunction. Identification of common variables (for example, those that come from better descriptions of human physiology and the dysregulated response to infection) across a variety of settings would enhance the ability to screen and treat the disease, and to study the effects of screening. Operationally, improved descriptions of relevant outcomes, and the linkage between screening tools, therapeutic responses to the screens and outcomes would help guide clinical recommendations.

Tools using data from the electronic medical records might, using algorithms, be adapted to predict cases of sepsis. There is a need to explore how algorithms could trigger appropriate diagnostic and therapeutic measures. The relevance of these algorithms may depend on the resources available and it will be important to assess whether they hold promise in lower income settings. Finding better ways to use the data available to clinicians in a variety of settings is an important focus for future research. 


\section{Important research questions:}

-What markers identify a dysregulated host response to infection?

- How can screening tools lead to actions that improve clinical outcomes?

- How can screening be implemented in resource-limited environments?

\section{How do we identify septic shock?}

Shock has long been viewed as an extreme form of circulatory insufficiency whose association with mortality is validated [123-125]. The entity is often classified based on etiology. In most cases, the clinical characteristic that identifies shock is hypotension. Septic shock is different; its defining characteristics involve more complex biology, which primarily reflects altered cellular function, aberrant metabolism or deranged biochemistry. Identification is based more on the recognition that normally adaptive, evolutionarily conserved mechanisms either become maladaptive or fail altogether. The rationale for defining septic shock as a unique entity appears to arise from two considerations. The first reflects the need for a syndrome analogous to other forms of circulatory insufficiency. In the articles generated by the first two sepsis consensus conferences [52, 105], septic shock was loosely defined as "sepsis with hypotension". The second involves outcome; numerous studies indicated that mortality was higher when sepsis was accompanied by hypotension.

Characterizing shock of any etiology becomes problematic when trying to determine how low the blood pressure must be for an entity to be called "shock". In most forms, the blood pressure is deemed to be sufficiently low to be considered shock if it is associated with a clinically apparent change in organ function, e.g., an altered mental status. Other characteristics used to identify organ dysfunction include oliguria, nausea/vomiting or shortness of breath. In sepsis, organ dysfunction often precedes the development of hypotension. By definition, it has to manifest in order to diagnose sepsis. Thus, conventional criteria for identifying shock may not apply. Indeed, it is tempting to consider "septic shock" as simply "sepsis where the sepsis-induced organ dysfunction lies in the cardiovascular system". An additional difficulty arises when considering the underlying cause of hypotension. In most forms of shock, the primary defect is known. This knowledge identifies a rational approach to therapy. In sepsis, however, the underlying defect is unknown. Indeed, virtually all the abnormalities that lead to hypotension in other shock states are present in sepsis (Table 2)-but these abnormalities are present whether or not there is hypotension.

To revise the definition of septic shock the Sepsis-3 task force sought to reconcile these different considerations. Given that sepsis-associated mortality is affected by what organ systems are dysfunctional, considering septic shock as a unique entity might not be justified. The view of shock as a state of cellular, and not organ system, dysfunction removed the requirement for hypotension. After debate, the task force arrived at a compromise. Thus, septic shock is defined as "a subset of sepsis in which underlying circulatory and cellular/metabolic abnormalities are profound enough to substantially raise mortality". This formulation encompasses both circulatory and cellular abnormalities 
while acknowledging that, at this time, the main distinction between sepsis and septic shock lies in the rate of survival.

\section{What is known}

In Sepsis-1, septic shock is defined as "sepsis with hypotension, despite adequate fluid resuscitation, along with the presence of perfusion abnormalities that may include but are not limited to, lactic acidosis, oliguria, or an acute alteration in mental status"[105]. Sepsis-2 defines septic shock as "a state of acute circulatory failure characterized by persistent arterial hypotension unexplained by other causes". The key criterion is hypotension "unexplained by other causes" [53]. Importantly, these "definitions" are really clinical criteria by which septic shock in patients might be recognized. Both Sepsis-1 and Sepsis-2 define hypotension as a systolic BP $<90 \mathrm{mmHg}$; Sepsis- 2 adds a mean $\mathrm{BP}<60 \mathrm{mmHg}$. Both sets of criteria are problematic. The choice of $90 \mathrm{mmHg}$ is arbitrary and neither measure provides an approach to assure that hypovolemia is absent. Lactate in sepsis may be elevated in the absence of hypotension, reflecting a sepsis-induced defect in oxidative phosphorylation ("aerobic glycolysis") or resulting from administered or endogenous catecholamines. Mental status changes are among the earliest observed clinical criteria for sepsis and may be present in the absence of septic shock. Oliguria is often an adaptive response to compensate for circulatory inadequacy and thus is a poor surrogate for renal dysfunction.

Perhaps the most important issue, however, is that both Sepsis-1 and Sepsis-2 are products of expert opinion. The choice of the variables used as clinical criteria (e.g., $\mathrm{SBP}<90 \mathrm{mmHg}$, lactic acidosis, oliguria, etc.) was made by task force members.

The clinical criteria for septic shock enumerated in Sepsis-3 were identified based on expert opinion, but the process was more refined. A literature search identified 44 studies reporting septic shock mortality that contributed to a list of the clinical criteria for septic shock. This list was used to inform a Delphi process among the task force members [54, 102]. The task force members chose hypotension, vasopressor therapy and hyperlactatemia as criteria for septic shock. These criteria were then applied to data from patients entered into the Surviving Sepsis Campaign (SSC) database who were designated as "having adequate fluid resuscitation, met SIRS criteria and had dysfunction in one or more organ systems". Results informed a second round of Delphi deliberations and approval of the need for vasopressors as a proxy for hypotension after resuscitation. A combination of all three variables identified cohorts of patients whose mortality was significantly higher than cohorts identified by any single variable or any combination of two variables, and were confirmed in cohorts of patients with suspected infection derived from two electronic health record (EHR) databases (54 and 35\% mortality) [126].

Since the publication of Sepsis-3, a limited number of studies examining septic shock have confirmed the findings [127]. The Sepsis-3 septic shock criteria were confirmed in a lower income population using data from a public hospital in Brazil [128] and in ICUs in Asia [129-132]. Sepsis-3 criteria have been successfully applied to children [133]. Stratification using lactate levels may improve accuracy [134]. Several studies have examined the potential contribution of criteria beyond hypotension, vasopressor use and lactate level, including a variety of biomarkers [124-133, 135-139]. In contrast to the Sepsis-3 
Table 2 Abnormalities leading to hypotension in various shock states, including septic shock

\begin{tabular}{|c|c|c|c|c|c|}
\hline Shock type & Venous return & Pump function & Vascular tone & $\begin{array}{l}\text { Other } \\
\text { abnormalities }\end{array}$ & Examples \\
\hline Cardiogenic & Normal or $\uparrow$ & $\downarrow \downarrow$ & Normal or $\uparrow$ & $\begin{array}{l}\text { Primary pump } \\
\text { failure, may } \\
\text { develop com- } \\
\text { pensatory salt/ } \\
\text { water retention } \\
\text { and increased } \\
\text { vascular tone }\end{array}$ & $\begin{array}{l}\text { Myocardial infarc- } \\
\text { tion, injury }\end{array}$ \\
\hline Neurogenic & $\downarrow$ & $\downarrow \downarrow$ & $\downarrow \downarrow$ & $\begin{array}{l}\text { Loss of inotropic/ } \\
\text { chronotropic } \\
\text { input and vascu- } \\
\text { lar tone }\end{array}$ & $\begin{array}{l}\text { High cervical } \\
\text { transection, spinal } \\
\text { anesthetic }\end{array}$ \\
\hline Distributive & $\downarrow \downarrow$ & $\begin{array}{l}\text { Normal (may } \uparrow \text { or } \\
\downarrow \text {, depending } \\
\text { on etiology and } \\
\text { compensation) }\end{array}$ & $\downarrow$ & $\begin{array}{l}\text { Mediators dilate a } \\
\text { vascular tree that } \\
\text { cannot be filled } \\
\text { with the existing } \\
\text { blood volume }\end{array}$ & $\begin{array}{l}\text { Anaphylaxis, adrenal } \\
\text { insufficiency, SIRS } \\
\text { response }\end{array}$ \\
\hline Septic & $\downarrow \downarrow$ & $\begin{array}{l}\uparrow \text { or normal, but } \\
\text { can be } \downarrow \downarrow\end{array}$ & $\downarrow \downarrow$ & $\begin{array}{l}\text { Dysfunctions in } \\
\text { 1. Vascular tone } \\
\text { leading to pool- } \\
\text { ing of venous } \\
\text { blood, } \\
\text { 2. Myocardial } \\
\text { pumping } \\
\text { 3. Vascular tone, } \\
\text { contributing to } \\
\text { hypotension }\end{array}$ & $\begin{array}{l}\text { Peritonitis, pneumo- } \\
\text { nia, urosepsis }\end{array}$ \\
\hline
\end{tabular}

Key abnormalities are indicated by bold double arrow

criteria for septic shock, the difficulty in obtaining measurement of most of these putative biomarkers limits their routine use (Table 2).

In perhaps the most comprehensive study to date, data from 205,632 patients identified as having sepsis using either Sepsis-1/2 or Sepsis-3 criteria were extracted from the national critical care database in England [51]. In this report, 92\% of patients met both Sepsis-1/2 and Sepsis-3 criteria for sepsis. In contrast, among 153,623 patients identified as having septic shock, only 38,896 patients (25\%) met both sets of criteria. Sepsis-3 criteria identified fewer $(39,262$ vs. 153,257$)$ patients with septic shock than Sepsis-1/2 criteria. Less than $1 \%$ of the patients identified by Sepsis-3 criteria did not satisfy Sepsis-1/2 criteria, suggesting better discrimination of a high-mortality cohort. ICU mortality among patients meeting Sepsis-3 criteria for septic shock was $47 \%$, significantly higher that the $22 \%$ rate among patients meeting Sepsis- 2 criteria for sepsis. Conversely, ICU mortality among patients meeting Sepsis-1/2 criteria for septic shock was $26 \%$. Importantly, all patients meeting Sepsis-3 criteria for septic shock had to meet criteria for sepsis, and most met both Sepsis-1/2 and Sepsis-3 criteria. As therapeutic approaches are the same for sepsis and septic shock patients, better discrimination need not come at the expense of missed cases.

Key points:

- Criteria for septic shock were produced by a consensus process and are nonspecific.

- Sepsis-3 criteria for septic shock reflect a more refined process and perform better at discriminating a high-mortality cohort while missing few cases. 


\section{What we do not know/gaps in knowledge}

The most readily explored open questions include validation of established criteria and evaluation of hemodynamics. Septic shock variables, alone or in combination, have not been verified prospectively. Areas for study include prospective validation of Sepsis-3 clinical criteria for septic shock, further evaluation of the criteria in resource challenged populations and environments, and investigation of the use of serum lactate levels to improve discrimination. The impact of added proxies that involve non-routine and potentially expensive tools (e.g., complex technologies, lab tests) on the validity of Sepsis-3 criteria is unknown. It is important to examine the cost-benefit ratio of any added criteria. Other clinical questions include: When, relative to fluid administration, should vasopressors be initiated? and: Is there a better "vasopressor of choice" than norepinephrine?

It remains unclear if there are tangible pathobiological difference between sepsis and septic shock. Such a dichotomy would demonstrate that septic shock is an entity distinct from sepsis and not just "sepsis with CV dysfunction". It also would eliminate mortality from the definition. Septic shock should be more than merely a subset of sepsis where mortality is substantially increased. In the current state of knowledge, we lack a "gold standard" that can be used to evaluate clinical criteria, biomarkers and other proxies. If septic shock is indeed a unique disorder, are there biomarkers that identify it without also identifying sepsis? If so, does it require treatment that is different from what is used to treat sepsis and support the circulation? Answers to these questions would support the clinical use of the term "septic shock".

Regarding the concept of shock, a better characterization of pathobiology could inform diagnosis and therapeutics. Is shock a macroscopic or microscopic phenomenon? Is there a spectrum - from biochemical to sub-cellular (e.g., mitochondrial) to cellular to tissue/organ system to systemic manifestations (e.g., hypotension, hyperthermia)? Are there ways to differentiate between these possibilities? In other forms of shock, the initiator is known.

Another unanswered question is whether there is a chronological progression from infection to sepsis to septic shock, or if it is possible to transition from infection directly to septic shock. This should be the case if the underlying pathobiology of sepsis and septic shock are different.

\section{Future directions}

New definitions or identifying criteria would be expected to disentangle "septic shock" from "sepsis with dysfunction of the cardiovascular system". If septic shock is to be a separate entity, the diagnostic approach to it should differ from that used for sepsis absent septic shock and efforts should seek to progress towards a "gold standard" for diagnosis and study. Obtaining insight as to whether underlying factors mediate the transition from simple infection to sepsis or to septic shock and justify designating septic shock as a unique entity is fundamental. Future research would specify therapeutic targets for septic shock that are independent of those attacked when treating sepsis. 


\section{Important research questions:}

- Is septic shock a distinct entity from sepsis?

- What is the pathobiology of septic shock?

- How can the presence of shock be confirmed independent of the diagnosis of sepsis?

- When should specific interventions be initiated in the setting of shock that would not otherwise be used in sepsis without shock?

\section{What in-hospital clinical information is associated with important outcomes in patients with sepsis?}

Treating sepsis should improve clinical outcomes, but addressing what constitutes an important outcome is a challenge. Viable construct criteria for sepsis should associate with whatever outcomes are selected. Ideally, treating some or all criteria improves these outcomes, but this relationship cannot be assumed. The presumed pathobiology of sepsis suggests that most criteria serve as markers rather than drivers of disease. These criteria should have clinically relevant outcomes validity. Whereas pursuit of a definition might improve the description of what sepsis is, clinical needs focus more on the parameters that can trigger therapies that improve outcomes.

Absent defining features, sepsis is either inferred subjectively (i. e., "I know it when I see it" [140]) or approximated by objective, imperfect proxies. Recognizing and appropriately treating a dysregulated host response to infection should be good clinical care, but to advance beyond encouraging vigilance and clinical acumen, investigations must uncover better indicators, links between these and sepsis, or find clinical signals for sepsis itself. Although a gold-standard signal is a laudable goal, it is most likely that science will continue to work through proxies.

\section{What is known}

The current identification paradigm for sepsis (Sepsis-3) makes use of established criteria (SOFA) [54]. This approach leverages a scoring system in the context of infection to provide a way to identify a population likely to benefit from therapies. This process is pragmatic but flawed. The SOFA score is twenty five years old and based on values and therapies that are in some cases outdated and are not themselves ideal criteria for organ or cellular dysfunction. There are many causes of hypoxia, altered mental status, elevated bilirubin or thrombocytopenia, each with different implications for a patient that might have sepsis. Sepsis-3 criteria are validated to mortality and ICU length of stay. However, neither mortality nor length of stay fully correlate to sepsis. Each is influenced by many other factors.

There are three essential elements to the concept of sepsis: infection, a dysregulated host response, and organ (cellular) dysfunction. Defining infection might be the least problematic, but it is imperfect. Defining a dysregulated host response implies a sound understanding of a regulated host response, but the understanding is incomplete. A stress response to injury has multiple descriptions [141, 142]. It is clear that the integrated and coordinated interactions of immune, $[143,144]$ neurologic, $[145,146]$ and 
endocrine systems [147] produce a series of physiologic adaptations [148]. Scoring systems attempt to describe organ dysfunctions. Although imperfect, they can provide a global index of dysfunction. Injury markers might improve this distinction [149].

Use of proxies leads to a compounding problem. As one proxy for organ dysfunction is combined with others, and then attributed as a part of a dysregulated host response resulting from infection, inaccuracies of each proxy multiply, such that the model's performance is degraded even if each component by itself is reasonably accurate. Linking proxies and outcomes, one sees a related problem. Assume a marker picks up 90\% of patients with sepsis. We want to correlate this marker with an outcome like ICU length of stay. If $90 \%$ of patients with sepsis have an increased length of stay, only $81 \%$ of those selected by the marker will have the outcome we are using to validate our model.

Sepsis does not occur in a clinical vacuum, and patient comorbidities, susceptibilities and responses (regulated and dysregulated) are expected to vary. A neutropenic or immunosuppressed patient will have a different response to bacteremia than someone with "normal" immune function. It may not be one disorder. Data suggest that there may be different phenotypes or endotypes for sepsis [75].

Outcomes validity for any scoring model is sensitive to the outcomes chosen. Sepsis-3 criteria use mortality and intensive care unit (ICU) stay of 3 days or longer [54], and many subsequent investigations have used similar outcomes to validate scoring systems. The mortality outcome presents a problem, as the goal is to identify patients that can be treated for sepsis. Improved performance of a model against mortality risks making the model a better mortality predictor or a triage tool. If a scoring system predicts mortality with $100 \%$ accuracy, it risks being irrelevant to clinicians hoping to improve survival.

Key points:

- Sepsis-3 criteria leverage a scoring system to identify patients likely to benefit from sepsis therapies.

- Most clinical information guiding the diagnosis of sepsis depends on proxies that may be markers rather than drivers of the dysregulated host response.

- Sepsis-3 criteria are validated against outcomes that may not be directly related to sepsis.

\section{What is not known/gaps in knowledge}

Available data inconclusively describe the relative beneficial and harmful effects of biologic perturbations, blurring the definitions of regulated and dysregulated. Clinical information consists of inflammatory biomarkers, endocrine abnormalities, altered metabolic signals and evidence of organ dysfunction, but the significance of each of these findings is unclear. Constructing a formulation of sepsis based on proxies doesn't necessarily specify the direction of the abnormality. For example, elevated stimulating hormones might reflect central overstimulation or peripheral underactivity, requiring different treatments. Clinicians and scientists struggle to separate dysfunctions driving a dysregulated host response from those caused by the host response. Available scoring systems lack organ-specificity. Improvements from the addition of data need further validation. 
We still know very little about which patterns in clinical information can identify patients requiring specific care to improve outcomes. Gaps include separating drivers from markers, injury from dysfunction, identifying infection and finding better characteristics for regulated and dysregulated host responses. We lack sufficient grasp of what should be important outcomes. Clinical research depends on uncovering patterns that identify patients at risk for clinically relevant outcomes and for whom interventions can change clinical trajectories. Available markers approximate interactions at a cellular and intracellular level. These proxies have yet to be mapped in a way that enables characterization of the whole organism response. We lack sufficient insight into model performance in both clinical construct validity (does the formulation approximate sepsis?) and outcome validity (does the formation accurately predict clinically meaningful outcomes?).

Patterns in response may be helpful ways to improve models and link them to valid outcomes, but key elements of these patterns are unexplained. In the example of the direction problem, sampling a panel of hormone function (e.g., a thyroid panel), rather than a single value (e.g., a thyroid stimulating hormone level), helps specifiy what the abnormality means. This is not as clear with sepsis. Recognizing patterns of clinical improvement or deterioration validates clinical suspicions. This approach only works if mechanisms are understood, an ongoing limitation. Efforts to quantify intensity [150] or clinical trajectory [151] add information to single-measurement proxies. Host response can vary in sepsis, and this variability provides useful staging information (152) and may help describe host response phenotypes (e.g., acute versus chronic critical illness) that shape clinical scoring tools and link them to outcomes, but these approaches are still speculative, requiring validation.

\section{Future directions}

Investigations into isolated organ dysfunctions would inform characterizations that could then be assessed in terms of causality, providing much needed insight into the mechanisms of sepsis. A focus on cellular dysfunction rather than nonspecific markers may help validate what relevant criteria establish a diagnosis whose treatment would be expected to improve outcomes. Further inquiry should explore clinical outcomes that approximate what a clinician would hope to improve by treating sepsis, such as by reversing organ dysfunctions (when they can be measured) or their effects.

Investigating the long-term effects and trajectories of organ dysfunctions could inform discussions about their nature and meaning. Interactions and more subtle data patterns (e.g., heart rate variability, pairing autonomic, endocrine and immune signals) can be investigated and may provide insight about integrated host responses to infection. How patient factors influence clinical data outcomes prediction deserves further study. The interactions of neurologc, endocrine and immune systems with critical functions such as metabolism, coagulation, and cell death, need characterization. Investigations should aim to build mechanistic models that help explain how complementary clinical data relate to each other and describe the benefits and harms of adaptation patterns. Finally, future investigations should address clinical criteria construct validitiy and outcome validity. 


\section{Important research questions:}

- How can markers of a dysregulated host response be distinguished from drivers of that response?

- How do cellular-level processes interact to produce organism-level responses?

- What is the best way to characterize a dysregulated response to infection, optimizing clinical criteria, construct validity and outcomes validity?

\section{Conclusions/summary}

The six questions addressed by this paper identify important issues about how to define, categorize, assess, predict, diagnose and treat sepsis. Subcommittee members, representing a broad group of clinicians from different parts of the world, addressed these considerations from a variety of perspectives. However, although the process used the common Delphi standard, it is based on the consensus of experts and is therefore subjective and prone to bias.

The accounts of what is known, the identified knowledge gaps, and the future directions for research serve as an important and prioritized resource for future sepsis investigations. Addressing these key gaps will lead to better understanding of disease epidemiology, pathobiology and individual patient heterogeneity, which will facilitate timely and accurate sepsis patient identification to deliver effective therapies, building on the progress made by the Sepsis-3 Task Force and the Surviving Sepsis Campaign.

Acknowledgements

The authors would like to acknowledge the European Society of Critical Care medicine and the Society of Critical Care Medicine for their support of the Surviving Sepsis Campaign Research Committee. Committee members include: Massimo Antonelli, MD; Judith Hellman, MD; Sameer Jog, MD, IDCCM; Jozef Kesecioglu, MD, PhD; Ishaq Lat, PharmD; and Mitchell M. Levy, MD, MCCM.

\section{Authors' contributions}

MEN: wrote portions of the manuscript, edited the entire manuscript and approved of the final draft. RF: wrote portions of the manuscript, and approved of the final draft. GSM: wrote portions of the manuscript, and approved of the final draft. IM-L: wrote portions of the manuscript, and approved of the final draft. FRM: wrote portions of the manuscript, and approved of the final draft. DDB: wrote portions of the manuscript, and approved of the final draft. CMC: wrote portions of the manuscript, and approved of the final draft. CSD: wrote portions of the manuscript, edited the entire manuscript and approved of the final draft. All authors read and approved the final manuscript.

Funding

The authors volunteered their time to producing this manuscript and no funding was used to produce it.

\section{Availability of data and materials}

Supporting data are cited in this narrative review. Mark E. Nunnally: no competing interests. Ricard Ferrer: Dr. Ferrer is a paid Board member for Merck Sharp and Dohme, and Shionogi, has received a grant from Becton, Dickinson and Company, has received payment for development of educational presentations from Pfizer, Grifols, Thermo and Baxter, and owns stock or stock options with Grifols. Greg S. Martin: no competing interests. Ignacio Martin-Loeches: Dr. MartinLoeches serves on advisory boards with Gilead, Merck, Pfizer and Accelerate Diagnostics. Flavia R. Machado: no competing interests. Daniel De Backer: Dr. DeBacker is past president of the European Society of Intensive Care Medicine and has received consulting fees from Fresenius Kabi. Craig M. Coopersmith: no competing interests. Clifford S. Deutschman: Dr. Deutschman reports grants from the Nationa Institute of General Medical Sciences, during the preparation of the manuscript; stock options from Enlivex, study drug supply from La Jolla Pharmaceuticals, and personal fees from Society of Critical Care Medicine, Pfizer and Sage Therapeutics, outside the submitted work.

\section{Declarations}

Ethics approval and consent to participate

This manuscript is a narrative review of published research and no ethics approval was sought. There are no study subjects and no consent process was necessary. 


\section{Consent for publication}

All authors consent to publication and there are no study subjects.

\section{Competing interests}

The authors provided the following information regarding competing interests.

\section{Author details}

${ }^{1}$ New York University School of Medicine, New York, NY, USA. ${ }^{2}$ Intensive Care Department, Vall d'Hebron University Hospital, Barcelona, Spain. ${ }^{3}$ Shock, Organ Dysfunction and Resuscitation (SODIR) Research Group, Vall d'Hebron Institut de Recerca, Barcelona, Spain. ${ }^{4}$ Division of Pulmonary, Allergy, Critical Care and Sleep Medicine, Department of Medicine, Grady Memorial Hospital and Emory Critical Care Center, Emory University, Atlanta, GA, USA. ${ }^{5}$ Multidisciplinary Intensive Care Research Organization (MICRO), Department of Intensive Care Medicine, St. James's University Hospital, Trinity Centre for Health Sciences, Dublin, Ireland. ${ }^{6}$ Hospital Clinic, IDIBAPS, Universidad de Barcelona, CIBERes, Barcelona, Spain. ${ }^{7}$ Universidade Federal de São Paulo, Sao Paulo, Brazil. ${ }^{8} \mathrm{Chirec} \mathrm{Hospitals,} \mathrm{Université} \mathrm{Libre} \mathrm{de} \mathrm{Bruxelles,} \mathrm{Brussels,} \mathrm{Belgium.}$ ${ }^{9}$ Department of Surgery and Emory Critical Care Center, Emory University, Atlanta, GA, USA. ${ }^{10}$ Department of Pediatrics, Cohen Children's Medical Center, Northwell Health, New Hyde Park, NY, USA. ${ }^{11}$ The Feinstein Institute for Medical Research/ Elmezzi Graduate School of Molecular Medicine, Manhasset, NY, USA.

Received: 4 February 2021 Accepted: 7 June 2021

Published online: 02 July 2021

\section{References}

1. Rhodes A, Evans LE, Alhazzani W et al (2017) Surviving Sepsis Campaign: international guidelines for management of sepsis and septic shock: 2016. Crit Care Med 45(3):486-552

2. Coopersmith CM, De Backer D, Deutschman CS et al (2018) Surviving sepsis campaign: research priorities for sepsis and septic shock. Intensive Care Med 44:1400-1426

3. Deutschman CS, Hellman J, Roca RF et al (2020) The surviving sepsis campaign: basic/translational science research priorities. Intensive Care Med Exp 8(1):31

4. Lat I, Coopersmith CM, De Backer D et al (2021) The surviving sepsis campaign: fluid resuscitation and vasopressor therapy research priorities in adult patients. Intensive Care Med Exp 9(1):10

5. Coopersmith CM, Antonelli M, Bauer SR et al (2021) The Surviving Sepsis Campaign: research priorities for coronavirus disease 2019 in critical illness. Crit Care Med 49(4):598-622

6. Hwang SY, Shin J, Jo IJ et al (2019) Delayed antibiotic therapy and organ dysfunction in critically ill septic patients in the emergency department. J Clin Med 8:222

7. Gaieski DF, Mikkelsen ME, Band RA et al (2010) Impact of time to antibiotics on survival in patients with severe sepsis or septic shock in whom early goal-directed therapy was initiated in the emergency department. Crit Care Med 38:1045-1053

8. Liu VX, Fielding-Singh V, Greene JD et al (2017) The timing of early antibiotics and hospital mortality in sepsis. Am J Respir Crit Care Med 196:856-863

9. Seymour CW, Gesten F, Prescott HC et al (2017) Time to treatment and mortality during mandated emergency care for sepsis. N Engl J Med 376:2235-2244

10. Johnston ANB, Park J, Doi SA et al (2017) Effort of immediate administration of antibiotics in patients with sepsis in tertiary care: a systematic review and meta-analysis. Clin Ther 39:190-202.e6

11. Vincent $J \mathrm{~L}$ (2016) The clinical challenge of sepsis identification and monitoring. Plos Med 13:e1002022

12. Filbin MR, Lynch J, Gillingham TD et al (2018) Presenting symptoms independently predict mortality in septic shock: importance of a previously unmeasured confounder. Crit Car Med 46:1592-1599

13. Plata-Menchaca EP, Ferrer R (2018) Life-support tools for improving performance of the Surviving Sepsis Campaign hour-1 bundle. Med Intensiva 42:547-550

14. Prescott HC, Cope TM, Gesten FC et al (2018) Reporting of sepsis cases for performance measurement versus for reimbursement in New York state. Crit Care Med 46:666-673

15. Kim M, Song KM, Kim CJ et al (2016) Electronic alerts with automated consultations promote appropriate antimicrobial prescriptions. PLOS ONE 11:0160551

16. Miller RR 3rd, Dong L, Nelson NC et al (2013) Multicenter implementation of a severe sepsis and septic shock treatment bundle. Am J Respir Crit Care Med 188:77-82

17. Armen SB, Freer CV, Showalter JW et al (2016) Improving outcomes in patients with sepsis. Am J Med Qual 31:56-63

18. Ferrer R, Artigas A, Levy MM et al (2008) Improvement in process of care and outcome after a multicenter severe sepsis educational program in Spain. JAMA 299:2294-2303

19. Jeon K, Shin TG, Sims MS et al (2012) Improvements in compliance with resuscitation bundles and achievement of end points after an educational program on the management of severe sepsis and septic shock. Shock 37:463-467

20. Girardis M, Rinaldi L, Donno L et al (2009) Effects on management and outcome of severe sepsis and septic shock patients admitted to the intensive care unit after implementation of a sepsis program: a pilot study. Crit Care 13:R143

21. Bloos $F$, Ruddel $H$, Thomas-Ruddel $D$ et al (2017) Effect of a multifaceted educational intervention for anti-infectious measures on sepsis mortality: a cluster randomized trial. Intensive Care Med 43:1602-1612

22. Noritomi DT, Ranzani OT, Monteiro MB et al (2014) Implementation of a multifaceted sepsis education program in an emerging country setting: clinical outcomes and cost-effectiveness in a long-term follow-up study. Intensive Care Med 40:182-191 
23. Ferrer R, Martinez ML, Goma G et al (2018) Improved empirical antibiotic treatment of sepsis after an educational intervention: the ABISS-Edusepsis study. Crit Care 22:167

24. Hranjec T, Sawyer RG (2013) Aggressive versus conservative initiation of antibiotics - authors'reply. Lancet Infect Dis 13:390

25. Alam N, Oskam E, Stassen PM et al (2018) Prehospital antibiotics in the ambulance for sepsis: a multicentre, open label, randomised trial. Lancet Respir Med 6:40-50

26. Rhodes A, Phillips G, Beale R et al (2015) The Surviving Sepsis Campaign bundles and outcome: results from the International Multicentre Prevalence Study on Sepsis (the IMPreSS study). Intensive Care Med 41:1620-1628

27. Cooke CR, Iwashyna TJ (2014) Sepsis mandates: improving inpatient care while advancing quality improvement. JAMA 312:1397-1398

28. Evans IVR, Phillips GS, Alpern ER et al (2018) Association between the New York Sepsis Care Mandate and inhospital mortality for pediatric sepsis. JAMA 320:358-367

29. Levy MM, Gesten FC, Phillips GS et al (2018) Mortality changes associated with mandated public reporting for sepsis. The results of the New York State initiative. Am J Respir Care Care Med 198:1406-1412

30. Barochia AV, Cui X, Vitberg D et al (2010) Bundled care for septic shock: an analysis of clinical trials. Crit Care Med 38:668-678

31. Berenson R, Pronovost $\mathrm{P}$, Krumholz $\mathrm{H}$. Achieving the potential of health care performance measures. Robert Woods Johnson Foundation and the Urban Institute; May, 2013. http://www.rwjf.org/en/research-publications/ find-rwjf-research/2013/05/achieving-the-potential-of-health-care-performance-measures.html and http://www urban.org/publications/412823.html.

32. Yebenes JC, Lorencio C, Esteban E et al (2020) Interhospital sepsis code in Catalonia (Spain): territorial model for initial care of patients with sepsis. Med Intnsiva 44:36-45

33. Walsh D, Gekle R, Bramante R et al (2020) Emergency department sepsis huddles: achieving excellence for sepsis benchmarks in New York State. Am J Emerg Med 38:222-224

34. Vermeir P, Vandijck D, Degroote $S$ et al (2015) Communication in healthcare: a narrative review of the literature and practical recommendations. Int J Clin Pract 69:1257-1267

35. Amaral AC, Fowler RA, Pinto R et al (2016) Patient and organizational factors associated with delays in antimicrobial therapy for septic shock. Crit Care Med 44:2145-2153

36. Kumar A, Roberts D, Wood KE et al (2006) Duration of hypotension before initiation of effective antimicrobial therapy is the critical determinant of survival in human septic shock. Crit Care Med 34:1589-1596

37. Dellinger RP, Carlet JRM, Gerlach $\mathrm{H}$ et al (2004) The surviving sepsis guidelines: not another "groundhog day." Crit Care Med 32:1601-1602

38. Levy MM, Artigas A, Phillips GS et al (2012) Outcomes of the Surviving Sepsis Campaign in intensive care units in the USA and Europe: a prospective cohort study. Lancet Infect Dis 12:919-924

39. Durbin J, Barnsley J, Finlayson B et al (2012) Quality of communication between primary health care and mental health care: an examination of referral and discharge letters. J Behav Health Serv Res 39:445-461

40. Epstein RM (1995) Communication between primary care physicians and consultants. Arch Fam Med 4:403-409

41. Kaelber DC, Bates DW (2007) Health information exchange and patient safety. J Biomed Inform 40:S40-45

42. Jiwa M, Dhaliwal S (2012) Referral writer: preliminary evidence for the value of comprehensive referral letters. Qual Prim Care 20:39-45

43. Coz Yataco A, Jaehne AK, Rivers EP (2017) Protocolized early sepsis care is not only helpful for patients: it prevents medical errors. Crit Care Med 45:464-472

44. Mao Q, Jay M, Hoffman JL et al (2018) Multicentre validation of a sepsis prediction algorithm using only vital sign data in the emergency department, general ward and ICU. BMJ Open 8:e017833

45. Goodwin M, Ito K, Gupta AH et al (2016) Protocolized care for early shock resuscitation. Curr Opin Crit Care 22:416-423

46. Polito CC, Isakov A, Yancey AH 2nd et al (2015) Prehospital recognition of severe sepsis: development and validation of a novel EMS screening tool. Am J Emerg Med 33:1119-1125

47. Baldira J, Ruiz-Rodriguez JC, Wilson DC et al (2020) Biomarkers and clinical scores to aid the identification of disease severity and intensive care requirement following activation of an in-hospital sepsis code. Ann Intensive Care 10:7

48. Long D, Capan M, Mascioli S et al (2018) Evaluation of user-interface alert displays for clinical decision support systems for sepsis. Crit Care Nurse 38:46-54

49. Westphal GA, Pereira AB, Fachin SM et al (2018) An electronic warning system helps reduce the time to diagnosis of sepsis. Rev Bras Ter Intensiva 30:414-422

50. Lilienfeld DE (1978) Definitions of epidemiology. Am J Epidemiol 107:87-90

51. Shankar-Hari M, Harrison DA, Rubenfeld GD et al (2017) Epidemiology of sepsis and septic shock in critical care units: comparison between Sepsis-2 and Sepsis-3 populations using a national critical care database. Br J Anaesth 119:626-636

52. Levy MM, Fink MP, Marshall JC et al (2003) 2001 SCCM/ESICM/ACCP/ATS/SIS international sepsis definitions conference. Crit Care Med 31:1250-1256

53. Abe T, Yamakawa K, Ogura H et al (2020) Epidemiology of sepsis and septic shock in intensive care units between sepsis-2 and sepsis-3 populations: sepsis prognostication in intensive care unit and emergency room (SPICE-ICU). J Intensive Care 8:44

54. Singer M, Deutschman CS, Seymour CW et al (2016) The third international consensus for sepsis and septic shock (Sepsis-3). JAMA 315:801-810

55. Rhee C, Dantes R, Epstein L et al (2017) Incidence and trends of sepsis in US hospitals using clinical vs claims data, 2009-2014. JAMA 318:1241-1249

56. Rudd KE, Johnson SC, Agesa KM et al (2020) Global, regional, and national sepsis incidence and mortality, 1990-2017: analysis for the Global Burden of Disease Study. Lancet 395:200-211

57. Kempker JA, Martin GS (2016) The changing epidemiology and definitions of sepsis. Clin Chest Med 37:165-179 
58. Martin GS, Mannino DM, Eaton S et al (2003) The epidemiology of sepsis in the United States from 1979 through 2000. N Engl J Med 348:1546-1554

59. Gaieski DF, Edwards JM, Kallan MJ et al (2013) Benchmarking the incidence and mortality of severe sepsis in the United States. Crit Care Med 41:1167-1174

60. Rhee C, Murphy MV, Li L et al (2015) Comparison of trends in sepsis incidence and coding using administrative claims versus objective clinical data. Clin Infect Dis 60:88-95

61. Klein Klouwenberg PM, Cremer OL, van Vught LA et al (2015) Likelihood of infection in patients with presumed sepsis at the time of intensive care unit admission: a cohort study. Crit Care 19:319

62. Beck MK, Jensen AB, Nielsen AB et al (2016) Diagnosis trajectories of prior multi-morbidity predict sepsis mortality. Sci Rep 6:36624

63. Ding $R$, Meng Y, Ma X (2018) The central role of the inflammatory response in understanding the heterogeneity of Sepsis-3. Biomed Res Int. https://doi.org/10.1155/2018/5086516

64. Leligdowicz A, Matthay MA (2019) Heterogeneity in sepsis: new biological evidence with clinical applications. Crit Care 23:80

65. Delano MJ, Ward PA (2016) The immune system's role in sepsis progression, resolution and long-term outcome. Immunol Rev 274:330-353

66. Meyer NJ, Reilly JP, Anderson BJ et al (2018) Mortality benefit of recombinant human interleukin-1 receptor antagonist for sepsis varies by initial interleukin-1 receptor antagonist plasma concentration. Crit Care Med 46:21-28

67. Pickkers P, van der Poll T (2019) What's new in immunostimulating strategies in the ICU. Intensive Care Med 45:110-112

68. Prescott HC, Calfee CS, Thompson BT et al (2016) Toward smarter lumping and smarter splitting: rethinking strategies for sepsis and acute respiratory distress syndrome clinical trial design. Am J Respir Crit Care Med 194:147-155

69. Torres A, Sibila O, Ferrer M et al (2015) Effect of corticosteroids on treatment failure among hospitalized patients with severe community-acquired pneumonia and high inflammatory response: a randomized clinical trial. JAMA 313:677-686

70. Pepper DJ, Sun J, Rhee C et al (2019) Procalcitonin-guided antibiotic discontinuation and mortality in critically ill adults: a systematic review and meta-analysis. Chest 155:1109-1118

71. Sarma A, Calfee CS, Ware LB (2020) Biomarkers and precision medicine: state of the art. Crit Care Clin 36:155-165

72. Davenport EE, Burnham KL, Radhakrishanan J et al (2016) Genomic landscape of the individual host response and outcomes in sepsis: a prospective cohort study. Lancet Respir Med 4:259-271

73. Scicluna BP, van Vught LA, Zwinderman AH et al (2017) Classification of patients with sepsis according to blood genomic endotype: a prospective cohort study. Lancet Respir Med 5:816-826

74. Sweeney TE, Perumanl TM, Henao R et al (2018) A community approach to mortality prediction in sepsis via gene expression analysis. Nat Commun 9:694

75. Seymour CW, Kennedy JN, Wang S et al (2019) Derivation, validation and potential treatment implications of novel clinical phenotypes for sepsis. JAMA 321:2003-2017

76. Sweeney TE, Azad TD, Donato M et al (2018) Unsupervised analysis of transcriptomics in bacterial sepsis across multiple datasets reveals three robust clusters. Crit Care Med 46:915-925

77. Rudd KE, Kissoon N, Limmathurotsakul D et al (2018) The global burden of sepsis: barriers and potential solutions. Crit Care 22:232

78. Wong HR (2017) Intensive care medication in 2050: precision medicine. Intensive Care Med 43:1507-1509

79. Stanski NL, Wong HR (2020) Prognostic and predictive enrichment in sepsis. Nat Rev Nephrol 16:20-31

80. Esper AM, Martin GS (2011) The impact of comorbid [corrected] conditions on critical illness. Crit Care Med 39:2728-2735

81. Esper AM, Moss M, Martin GS (2009) The effect of diabetes mellitus on organ dysfunction with sepsis: an epidemiological study. Crit Care 12:R18

82. Torres VB, Azevedo LC, Silva UV et al (2015) Sepsis-associated outcomes in critically ill patients with malignancies. Ann Am Thorac Soc 12:1185-1192

83. Vincent JL, Beumier M (2013) Diagnostic and prognostic markers in sepsis. Expert Rev Anti Infect Ther 11:265-275

84. Anderson BJ, Calfee CS, Liu KD et al (2019) Plasma sTNFR1 and IL8 for prognostic enrichment in sepsis trials: a prospective cohort study. Crit Care 23:400

85. Gibot S, Bene C, Noel R et al (2012) Combination biomarkers to diagnose sepsis in the critically ill patient. Am J Respir Crit Care Med 186:65-71

86. Herridge MS, Cheung AM, Tansey CM et al (2003) One-year outcomes in survivors of the acute respiratory distress syndrome. N Engl J Med 348:683-693

87. Herridge MS, Tansey CM, Matté A et al (2011) Functional disability 5 years after acute respiratory distress syndrome. N Engl J Med 364:1293-1304

88. Bagshaw SM, Uchino S, Bellomo R et al (2007) Septic acute kidney injury in critically ill patients: clinical characteristics and outcomes. Clin J Am Soc Nephrol 2:431-439

89. Ketteler M, Block GA, Evenepoel P et al (2018) Diagnosis, evaluation, prevention and treatment of chronic kidney disease-mineral and bone disorder: synopsis of the kidney disease: improving global outcomes 2017 clinical practice guidelines update. Ann Intern Med 168:422-430

90. ARDS Definition Task Fourse, Ranieri VM, Rubenfield GD, et al. Acute respiratory distress syndrome: the Berlin Definition. JAMA. 2012:307:2526-2533

91. Yaegashi Y, Shirakawa K, Sato N et al (2005) Evaluation of a newly identified soluble CD14 subtype as a marker for sepsis. J Infect Chemother 11:234-238

92. Shozushima T, Takahashi G, Matsumoto N et al (2011) Usefulness of presepsin (sCD14-ST) measurements as a marker for the diagnosis and severity of sepsis that satisfied diagnostic criteria of systemic inflammatory response syndrome. J Infect Chemother 17:764-769

93. Li S, Huang X, Chen Z et al (2013) Neutrophil DC64 expression as a biomarker in the early diagnosis of bacterial infection: a meta-analysis. Int J Infect Dis 17:e12-23 
94. Langley RJ, Tsalik EL, van Velkinburgh JC et al (2013) An integrated clinico-metabolomic model improves prediction of death in sepsis. Sci Transl Med 5:195ra95

95. Calfee CS, Delucchi KL, Sinha P et al (2018) Acute respiratory distress syndrome subphenotypes and differential response to simvastin: secondary analysis of a randomised controlled trial. Lancet Respir Med 6:691-698

96. Famous KR, Delucci K, Ware M et al (2017) Acute respiratory distress syndrome subphenotypes respond differently to randomized fluid management strategy. Am J Respir Crit Care Med 195:331-338

97. Sinha P, Delucchi KL, McAuley DF et al (2020) Development and validation of parsimonious algorithms to classify acute respiratory distress syndrome phenotypes: a secondary analysis of randomised controlled trials. Lancet Respir Med 8:247-257

98. Sinha P, Delucchi KL, Thompson BT et al (2018) Latent class analysis of ARDS subphenotypes: a secondary analysis of the statins for acutely injured lungs from sepsis (SAILS) study. Intensive Care Med 44:1859-1869

99. Calfee CS, Delucchi K, Parsons PE et al (2014) Subphenotypes in acute respiratory distress syndrome: latent class analysis of data from two randomised controlled trials. Lance Respir Med 2:611-620

100. Vincent JL, Moreno R, Rakala J et al (1996) The SOFA (Sepsis-related Organ Failure Assessment) score to describe organ dysfunction/failure. On behalf of the Working Group on Sepsis-Related Problems of the European Society of Intensive Care Medicine. Intensive Care Med 22:707-710

101. Le G Jr, Klar J, Lemeshow S et al (1996) The Logistic Organ Dysfunction system. A new way to assess organ dysfunction in the intensive care unit. ICU Scoring Group. JAMA 276:802-810

102. Seymour CW, Liu VX, Iwashyna TJ et al (2016) Assessment of clinical criteria for sepsis for the third international consensus definitions for sepsis and septic shock (Sepsis-3) [published correction appears in JAMA. 12016;315:2237]. JAMA 315:762-774

103. Vincent JL, de Mendoca A, Cantraine F et al (1998) Use of the SOFA score to assess the incidence of organ dysfunction/failure in intensive care units: results of a multicenter, prospective study. Working group on "sepsisrelated problems" of the European Society of Intensive Care Medicine. Crit Care Med 26:1793-1800

104. Calvano SE, Coyle SM, Barbosa KS et al (1998) Multivariate analysis of 9 disease-associated variables for outcome prediction in patients with sepsis. Arch Surg 133:1347-1350

105. Bone RC, Balk RA, Cerra FB et al (1992) Definitions for sepsis and organ failure and guidelines for the use of innovative therapies in sepsis. The ACCP/SCCM Consensus Conference Committee. American College of Chest Physicians/Society of Critical Care Medicine. Chest 101:1644-1655

106. Kaukonen K, Bailey M, Pilcher D et al (2015) Systemic inflammatory response syndrome criteria in defining severe sepsis. N Eng J Med 372:1629-1638

107. Baig MA, Sheikh S, Hussain E et al (2018) Comparison of qSOFA and SOFA score for predicting mortality in severe sepsis and septic shock patients in the emergency department of a low middle income country. Turk J Emerg Med 18:148-151

108. Williams JM, Greenslade JH, McKenzie JV et al (2017) Systemic inflammatory response syndrome, quick sequential organ function assessment, and organ dysfunction: insights from a prospective database of ED patients with infection. Chest 151:586-596

109. Anand V, Zhang Z, Kadri SS et al (2019) Epidemiology of quick sequential organ failure assessment criteria in undifferentiated patients and association with suspected infection and sepsis. Chest 156:289-297

110. Ranzani OT, Prina E, Menendez R et al (2017) New sepsis definition (Sepsis-3) and community-acquired pneumonia mortality. A validation and clinical decision-making study. Am J Respir Crit Care Med 196:1287-1297

111. Freund $Y$, Lemachatti N, Krastinova E et al (2017) Prognostic accuracy of Sepsis-3 criteria for in-hospital mortality among patients with suspected infection presenting to the emergency department. JAMA 317:301-308

112. Finkelsztein EJ, Jone DS, Ma KC et al (2017) Comparison of aSOFA and SIRS for predicting adverse outcomes of patients with suspicion of sepsis outside the intensive care unit. Crit Care 21:73

113. Rudd KE, Seymour CW, Aluisio AR et al (2018) Association of the Quick Sequential (sepsis-related) Organ Failure Assessment (qSOFA) score with excess hospital mortality in adults with suspected infection in low- and middleincome countries. JAMA 319:2202-2211

114. Rhee C, Zhang Z, Kadri SS, et al. Sepsis surveillance using adult sepsis events simplified eSOFA criteria versus Sepsis-3 Sequential Organ Failure Assessment criteria. Crit Care Med. 2019;47(3):307-14.

115. National Early Warning Score (NEWS): Standardising the assessment of acute-illness severity in the NHS. Report of a working party London: RCP, 2017. 77 https://www.rcplondon.ac.uk/file/8636/download. Accessed 23 June 2020

116. Subbe CP, Kruger M, Seymour CW et al (2001) Validation of a modified early warning score in medical admissions. QJM 94:521-526

117. Keep JW, Messmer AS, Sladden R et al (2016) National early warning score at Emergency Department triage may allow earlier identification of patients with severe sepsis and septic shock: a retrospective observational study. Emerg Med J 33:37-41

118. Usman OA, Usman AA, Ward MA (2019) Comparison of SIRS, qSOFA, and NEWS fo the early identification of sepsis in the Emergency Department. Am J Emerg Med 37:1490-1497

119. Schorr CA, Zanotti S, Dellinger RP (2014) Severe sepsis and septic shock: management and performance improvement. Virulence 5:190-199

120. Fleuren LM, Klausch TLT, Zwager CL et al (2020) Machine learning for the prediction of sepsis: a systematic review and meta-analysis of diagnostic test accuracy. Intensive Care Med 46:383-400

121. Henry KE, Hager DN, Pronovost RJ, Saria S (2015) A targeted real-time early warning score (TREScore) for septic shock. Sci Transl Med 7:299ra122

122. Nemati S, Holder A, Razmi F et al (2018) An interpretable machine learning model for accurate prediction of sepsis in the ICU. Crit Care Med 46:547-553

123. Nguyen HL, Yarzebski J, Lessard D et al (2017) Ten-year (2001-2011) trends in the incidence rates and short-term outcomes of early versus late onset cardiogenic shock after hospitalization for acute myocardial infarction. J Am Heart Assoc 6:E005566 
124. Sakr Y, Reinhart K, Vincent JL et al (2006) Does dopamine administration shock influence outcome? Results of the sepsis occurrence in acutely ill patients (SOAP) study. Crit Care Med 34:589-597

125. Parks JK, Elliott AC, Gentilello LM et al (2006) Systemic hypotension is a late marker of shock after trauma: a validation study of Advanced Trauma Life Support principles in a large national sample. Am J Surg 196:727-731

126. Shankar-Hari M, Phillips GS, Levy ML et al (2016) Developing a new definition and assessing new clinical criteria for septic shock: for the Third International Consensus Definitions for Sepsis and Septic Shock (Sepsis-3). JAMA 315:775-787

127. Fernando SM, Reardon PM, Rochwert B et al (2018) Sepsis-3 septic shock criteria and associated mortality among infected hospitalized patients assessed by a rapid response team. Chest 154:309-316

128. Besen BAMP, Romano TG, Nassar AP Jr et al (2016) Sepsis-3 definitions predict ICU mortality in a low-middleincome country. Ann Intensive Care 6:107

129. Ryoo SM, Kang GH, Shin TG et al (2018) Clinical outcome comparison of patients with septic shock defined by the new Sepsis-3 criteria and by previous criteria. J Thorac Dis 10:845-853

130. Takauji S, Hayakawa M, Fujita S (2019) A nationwide comparison between Sepsis-2 and Sepsis-3 definition in Japan. J Intensive Care Med. https://doi.org/10.1177/0885066618823151

131. Chen QH, Shao J, Liu WL et al (2019) Predictive accuracy of Sepsis-3 definitions for mortality among adult critically ill patients with suspected infection. Chin Med J 132:1147-1153

132. Kim JY, Kim HI, Suh GY et al (2019) Application of Sepsis-3 criteria to Korean patients with critical illnesses. Acute Crit Care 34:30-37

133. Matics TJ, Sanchez-Pinto LN (2017) Adaptation and validation of a pediatric sequential organ failure assessment score and evaluation of the Sepsis-3 definitions in critically ill children. JAMA Pediatr 1717:e172352

134. Ryoo SM, Lee JB, Lee YS et al (2018) Lactate level versus lactate clearance for predicting mortality in patients with septic shock defined by Sepsis-3. Crit Care Med 46:e489-e495

135. Hamed S, Behnes M, Pauly D et al (2017) Diagnostic value of Pentraxin-3 in patients with sepsis and septic shock in accordance with latest Sepsis-3 definitions. BMC Infect Dis 17:554

136. Barre M, Behnes M, Hamed S et al (2018) Revisiting the prognostic value of monocyte chemotactic protein 1 and interleukin-6 in the Sepsis-3 era. J Crit Care 43:21-28

137. Ljungstrom L, Pernestig AK, Jacobsson G et al (2017) Diagnostic accuracy of procalcitonin, neutrophil-lymphocyte count ration, C-reactive protean, and lactate in patients with suspected bacterial sepsis. PLoS ONE 12:e081704

138. Tian R, Wang X, Pan T et al (2019) Plasma PTX3, MCP1 and Ang2 are early biomarkers to evaluate the severity of sepsis and septic shock. Scand I Immunol 90:e1823

139. Song J, Park DW, Moon S et al (2019) Diagnostic and prognostic value of interleukin-6, pentraxin 3 and procalcitonin levels among sepsis and septic shock patients: a prospective controlled study according to the Sepsis-3 definitions. BMC Infect Dis 19:968

140. Lattman P. The origins of Justice Stewart's'I know it when I see it'. Wall Street J. LawBlog at The Wall Street Journal Online. 2007. Retrieved June 3, 2020.

141. Cuthbertson DP (1932) Observations on the disturbances of metabolism produced by injury of the limbs. QLM 1:233-235

142. Moore FD (1953) Bodily changes in surgical convalescence. I. The normal sequence observations and interpretations. Ann Surg 137:289-315

143. Hotchkiss RS, Tinsley KW, Swanson PE et al (2001) Sepsis-induced apoptosis causes progressive profound depletion of B and DC4+T lymphocytes in humans. J Immunol 166:6952-6963

144. Hotchkiss RS, Tinsley KS, Swanson PE et al (2020) Depletion of dendritic cells, but not macrophages, in patients with sepsis. J Immunol 168:2493-2500

145. Deutschman CS, Raj NR, McGuire EO, Kelz MB (2013) Orexinergic activity modulates altered vital signs and pituitary hormone secretion in experimental sepsis. Crit Care Med 41:e368-e375

146. Andersson U, Tracey KJ (2012) Reflex principles of immunological homeostasis. Annu Rev Immunol 30:313-335

147. Ingels C, Gunst J, Van den Berghe G (2018) Endocrine and metabolic alterations in sepsis and implications for treatment. Crit Care Clin 34:81-96

148. Godin P, Buchman T (1996) Uncoupling of biological oscillators. Crit Care Med 24:1107-1116

149. Han WK, Bailly V, Abichandani R et al (2002) Kidney injury molecule-1 (KIM-1): a novel biomarker for human renal proximal tubule injury. Kidney Int 62:237-244

150. Remick DG, Bolgos GR, Siddiqui J et al (2002) Six at six: interleukin- 6 measured $6 \mathrm{~h}$ after the initiation of sepsis predicts mortality over 3 days. Shock 17:463-467

151. Klein Klouwenberg PMC, Spitoni C, van der Poll T et al (2019) Predicting the clinical trajectory in critically ill patients with sepsis: a cohort study [published correction appears in Crit Care 2020;24:41]. Crit Care 23:408

152. Opal SM (2005) Concept of PIRO as a new conceptual framework to understand sepsis. Pediatr Crit Care Med 6:S55-S60

\section{Publisher's Note}

Springer Nature remains neutral with regard to jurisdictional claims in published maps and institutional affiliations. 